\title{
SPECTROSCOPY AND PHOTOMETRY OF NEARBY YOUNG SOLAR ANALOGS
}

\author{
E. J. GAIDOS ${ }^{1,2}$ \\ Jet Propulsion Laboratory, California Institute of Technology, 4800 Oak Grove Drive, Pasadena, CA 91109 \\ AND \\ G. W. Henry AND S. M. Henry \\ Center of Excellence in Information Systems, Tennessee State University, 330 Tenth Avenue North, Nashville, TN 37203-3401 \\ Received 2000 April 17; accepted 2000 May 5
}

\begin{abstract}
We present new photometry and spectroscopy of 34 stars from a catalog of 38 nearby $(d<25 \mathrm{pc}) \mathrm{G}$ and $\mathrm{K}$ dwarfs selected as analogs to the early Sun. We report that the least active star in our sample is also slowly rotating and probably of solar age. Two other stars appear to be evolved objects that have recently acquired angular momentum. A fourth star may be a spectroscopic binary. Many of the other stars belong to previously identified common proper-motion groups. Space motions, lithium abundances, and $\mathrm{Ca}$ II emission of these stars suggest ages between 70 and 800 Myr.
\end{abstract}

Key words: planetary systems - solar system: formation - stars: evolution - Sun

\section{INTRODUCTION}

Observations of young solar-type stars provide insight into the behavior of the Sun and its effect on the planets during the first few hundred million years of solar system history. Such clues are important in inferring early conditions on the Earth and other planets in the absence of an accessible geologic record. A catalog of 38 nearby stars was constructed for this purpose using published indicators of rapid rotation and high chromospheric activity as proxies of a young age (Gaidos 1998). Here we report results from the analysis of new spectroscopic and photometric data of the 34 stars observable from the northern hemisphere.

\section{OBSERVATIONS}

Differential photometry of 31 stars was acquired with two automatic photoelectric telescopes (APTs) located at Fairborn Observatory in southern Arizona. A $0.40 \mathrm{~m}$ APT (T3) observed 19 of the stars in the Johnson $B$ and $V$ bands, while a $0.75 \mathrm{~m}$ APT (T4) observed 12 stars in the Strömgren $b$ and $y$ bands. Details of the $0.40 \mathrm{~m}$ APT operation, data reduction procedures, and photometric precision can be found in Henry (1995). A complete discussion of photometry with the $0.75 \mathrm{~m}$ APT can be found in Henry (1999).

High-resolution $(R \sim 90,000)$ echelle spectra were obtained at the Kitt Peak 0.9 m coudé feed telescope with the $\mathrm{F} 5$ camera and $3 \mathrm{~K} \times 1 \mathrm{~K}$ Ford $\mathrm{CCD}$ during four observing runs (1998 May, July, and November, and 1999 March). Lower resolution $(R \sim 30,000)$ spectra in the region of the $\mathrm{Ca}$ II $\mathrm{H}$ and $\mathrm{K}$ lines were obtained in single-order grating mode. Typical total integration times were $\sim 1 \mathrm{hr}$ per star. Spectral images were bias subtracted and flattened with quartz lamp flat fields, and charged particle events were removed by median filtering of multiple exposures. Individual spectral orders were extracted, corrected for sky emission, and wavelength calibrated against thorium-argon arc spectra using the IRAF spectroscopic data reduction package.

\footnotetext{
${ }^{1}$ Visiting Astronomer, Kitt Peak National Observatory. KPNO is operated by the Association of Universities for Research in Astronomy, Inc., under cooperative agreement with the National Science Foundation.

${ }^{2}$ Visitor, Division of Geology and Planetary Science, California Institute of Technology.
}

\section{ANALYSIS}

Heliocentric radial velocities were computed through order-by-order comparison of echelle spectra pairs using the Fourier cross-correlation routine FXCOR in IRAF and averaging over many orders. Orders in which the crosscorrelation failed because of low stellar signal or the presence of strong telluric emission were excluded. Radial velocities were obtained for 25 solar analogs, including five previously lacking measurements in the literature. Statistical velocity uncertainties estimated using independently calibrated measurements of stars on different nights have a median value of $0.11 \mathrm{~km} \mathrm{~s}^{-1}$. With our few measurements we cannot distinguish between measurement errors and actual Doppler velocity variations for individual stars. Absolute velocities were calculated by comparison with published higher accuracy values on constant-velocity stars (Duquennoy \& Mayor 1991). Multiple velocity measurements of these stars exhibited similar variation, suggesting that the night-to-night differences are measurement errors. The space motions of 36 stars were calculated in righthanded Galactic coordinates with respect to the local standard of rest (LSR), using standard algorithms (Johnson \& Soderblom 1987) and solar motion $(+9.00,+11.53,+6.97)$ $\mathrm{km} \mathrm{s}^{-1}$ (Binney \& Tremaine 1987; Table 1). The stars have been divided into groups according to their space motions (see § 4). Uncertainties in space motion were calculated using the errors in parallax and proper motion reported in the Hipparcos catalog and with an uncertainty in radial velocity, if not known from multiple measurements, taken to be $0.11 \mathrm{~km} \mathrm{~s}^{-1}$. Our new radial velocity data, combined with previous observations, are sensitive to any variations exceeding $1 \mathrm{~km} \mathrm{~s}^{-1}$ on timescales of $\sim 1 \mathrm{yr}$ in 11 stars (Table 1). This is the reflex motion induced by an object of mass $m$ with $m \sin i<30$ Jovian masses on a 1 AU orbit, where $i$ is the orbital inclination.

The results of our photometric analysis of the APT data ${ }^{3}$ are found in Table 2. Many of the stars have observations over more than one observing season, and we analyzed each

\footnotetext{
${ }^{3}$ The individual observations are available on the Tennessee State University Automated Astronomy Group Web site at http:// schwab.tsuniv.edu/papers/aj/youngsuns/youngsuns.html.
} 
TABLE 1

SPACE Motions

\begin{tabular}{|c|c|c|c|c|c|c|c|}
\hline HD & $\begin{array}{c}\pi \\
\text { (mas) }\end{array}$ & $\begin{array}{c}\mu_{\alpha} \\
\left({\operatorname{mas~} \mathrm{yr}^{-1}}^{-1}\right)\end{array}$ & 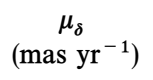 & $\begin{array}{c}v_{r} \\
\left(\mathrm{~km} \mathrm{~s}^{-1}\right)\end{array}$ & $\begin{array}{c}U \\
\left(\mathrm{~km} \mathrm{~s}^{-1}, \mathrm{LSR}\right)\end{array}$ & $\begin{array}{c}V \\
\left(\mathrm{~km} \mathrm{~s}^{-1}, \mathrm{LSR}\right)\end{array}$ & $\begin{array}{c}\quad W \\
\left(\mathrm{~km} \mathrm{~s}^{-1}, \mathrm{LSR}\right)\end{array}$ \\
\hline \multicolumn{8}{|c|}{ Ursa Major group: } \\
\hline $26923 \ldots \ldots \ldots$ & 47.20 & -109.4 & -108.3 & -7.4 & $23.2 \pm 0.2$ & $11.0 \pm 0.1$ & $-2.6 \pm 0.3$ \\
\hline $41593 \ldots \ldots \ldots$ & 64.71 & -122.3 & -103.3 & -10.1 & $19.7 \pm 0.1$ & $11.9 \pm 0.1$ & $-4.2 \pm 0.2$ \\
\hline $63433^{a} \ldots \ldots$ & 45.84 & -9.3 & -11.8 & -16.1 & $22.9 \pm 0.1$ & $14.0 \pm 0.1$ & $-1.0 \pm 0.1$ \\
\hline $72905^{a}$. & 70.07 & -27.7 & 87.9 & -12.7 & $18.9 \pm 0.1$ & $12.1 \pm 0.1$ & $-3.1 \pm 0.1$ \\
\hline $109011 \ldots \ldots$ & 42.13 & 99.7 & -13.2 & -13.1 & $23.1 \pm 0.8$ & $11.3 \pm 0.5$ & $-3.1 \pm 0.6$ \\
\hline $135559^{\mathrm{a}}$. & 64.19 & 178.0 & -136.5 & -3.1 & $18.7 \pm 0.2$ & $12.7 \pm 0.1$ & $-6.8 \pm 0.2$ \\
\hline $165185^{\mathrm{a}} \ldots \ldots$ & 57.58 & 105.4 & 8.0 & 14.9 & $23.0 \pm 0.1$ & $15.3 \pm 0.1$ & $-2.4 \pm 0.1$ \\
\hline \multicolumn{8}{|c|}{ Local association: } \\
\hline $166 \ldots \ldots \ldots$ & 72.98 & 379.9 & -178.3 & -7.0 & $-5.9 \pm 0.2$ & $-10.3 \pm 0.2$ & $-2.8 \pm 0.2$ \\
\hline $37394^{a} \ldots \ldots$ & 81.69 & 2.7 & -523.6 & 2.0 & $-5.4 \pm 1.1$ & $-11.4 \pm 0.5$ & $-6.9 \pm 0.3$ \\
\hline $97334^{\mathrm{a}}$. & 46.04 & -248.6 & -151.3 & -3.7 & $-6.7 \pm 0.3$ & $-11.7 \pm 0.5$ & $-4.1 \pm 0.2$ \\
\hline $116956^{\mathrm{a}}$. & 45.76 & -217.4 & 11.2 & -12.3 & $-7.0 \pm 0.3$ & $-6.9 \pm 0.2$ & $-1.1 \pm 0.1$ \\
\hline $206860^{\mathrm{a}}$. & 54.37 & 231.1 & -113.4 & -17.0 & $-5.6 \pm 0.2$ & $-10.1 \pm 0.1$ & $-3.8 \pm 0.3$ \\
\hline \multicolumn{8}{|c|}{ Possible Pleiades cluster: } \\
\hline $82443^{\mathrm{a}} \ldots \ldots$ & 56.35 & -147.5 & -246.3 & 8.2 & $-0.8 \pm 0.1$ & $-11.4 \pm 0.3$ & $1.6 \pm 0.2$ \\
\hline $113449^{b} \ldots \ldots$ & 45.20 & -189.8 & -219.6 & -11.0 & $-0.7 \pm 0.2$ & $-11.6 \pm 0.8$ & $-13.5 \pm 0.3$ \\
\hline \multicolumn{8}{|c|}{ Possible Hyades cluster: } \\
\hline $73350 \ldots \ldots \ldots$ & 42.32 & -298.4 & 43.3 & 35.1 & $-33.9 \pm 0.6$ & $-8.3 \pm 0.3$ & $-4.8 \pm 0.6$ \\
\hline $180161^{\mathrm{a}} \ldots \ldots$ & 50.00 & 217.8 & 408.3 & -27.1 & $-35.0 \pm 0.5$ & $-10.6 \pm 0.3$ & $-8.4 \pm 0.1$ \\
\hline \multicolumn{8}{|c|}{ High-velocity stars: } \\
\hline $152391^{\mathrm{a}} \ldots \ldots$ & 59.04 & -711.8 & -1483.7 & 44.9 & $94.0 \pm 0.7$ & $-98.8 \pm 1.8$ & $16.4 \pm 0.2$ \\
\hline $220182 \ldots \ldots$ & 45.63 & 636.2 & 219.2 & 3.0 & $-58.6 \pm 1.4$ & $-6.4 \pm 2.4$ & $4.8 \pm 0.7$ \\
\hline \multicolumn{8}{|l|}{ Old star: } \\
\hline $10780 \ldots \ldots \ldots$ & 100.24 & 582.0 & -246.8 & 2.3 & $-15.4 \pm 0.2$ & $-5.2 \pm 0.2$ & $2.0 \pm 0.1$ \\
\hline \multicolumn{8}{|l|}{ Others: } \\
\hline $1237 \ldots \ldots \ldots$ & 56.76 & 433.9 & -58.0 & $\ldots$ & $\ldots$ & $\ldots$ & $\ldots$ \\
\hline $1835 \ldots \ldots \ldots$ & 49.05 & 394.3 & 60.5 & -2.6 & $-26.7 \pm 0.7$ & $-3.3 \pm 0.3$ & $7.1 \pm 0.1$ \\
\hline $7590 \ldots \ldots \ldots$ & 42.30 & -112.4 & -30.5 & -13.4 & $27.4 \pm 0.2$ & $7.8 \pm 0.1$ & $6.9 \pm 0.1$ \\
\hline $10008 \ldots \ldots \ldots$ & 42.35 & 71.0 & -97.7 & 11.2 & $4.6 \pm 0.1$ & $0.2 \pm 0.3$ & $-5.7 \pm 0.1$ \\
\hline $11131 \ldots \ldots \ldots$ & 43.47 & -122.6 & -100.4 & -2.1 & $15.6 \pm 0.8$ & $-2.7 \pm 1.3$ & $14.4 \pm 0.8$ \\
\hline $20630^{a} \ldots \ldots$ & 109.18 & 268.9 & 93.5 & 18.9 & $-12.7 \pm 0.1$ & $7.1 \pm 0.1$ & $2.6 \pm 0.1$ \\
\hline $30495 \ldots \ldots \ldots$ & 75.10 & 130.4 & 169.2 & 21.7 & $-14.9 \pm 0.1$ & $2.9 \pm 0.1$ & $4.3 \pm 0.1$ \\
\hline $36435 \ldots \ldots \ldots$ & 51.10 & -148.9 & -92.8 & 12.9 & $18.4 \pm 0.1$ & $8.0 \pm 0.1$ & $-11.2 \pm 0.1$ \\
\hline $37394 \ldots \ldots \ldots$ & 81.69 & 2.7 & -523.6 & 2.0 & $-5.4 \pm 1.1$ & $-11.4 \pm 0.5$ & $-6.9 \pm 0.3$ \\
\hline $43162^{a} \ldots \ldots$ & 59.90 & -47.1 & 110.9 & 21.7 & $-11.3 \pm 0.1$ & $1.5 \pm 0.1$ & $0.1 \pm 0.1$ \\
\hline $52698 \ldots \ldots \ldots$ & 68.42 & 206.6 & 40.9 & 12.2 & $4.5 \pm 0.1$ & $-2.5 \pm 0.1$ & $19.0 \pm 0.2$ \\
\hline $59967 \ldots \ldots \ldots$ & 45.93 & -87.8 & 53.8 & 9.0 & $-2.3 \pm 0.1$ & $6.9 \pm 0.1$ & $0.2 \pm 0.1$ \\
\hline $72760 \ldots \ldots$ & 45.95 & -194.3 & 23.4 & 34.5 & $-26.0 \pm 0.3$ & $-7.8 \pm 0.1$ & $5.5 \pm 0.3$ \\
\hline $118972 \ldots \ldots$ & 64.08 & 205.9 & -166.0 & -7.3 & $17.9 \pm 0.2$ & $19.7 \pm 0.1$ & $-10.1 \pm 0.2$ \\
\hline $128400^{c} \ldots \ldots$ & 49.15 & 120.4 & -19.4 & $\ldots$ & $\ldots$ & $\ldots$ & $\ldots$ \\
\hline $128987^{\mathrm{a}} \ldots \ldots$ & 42.43 & -111.9 & -65.2 & -23.0 & $-13.4 \pm 0.2$ & $4.9 \pm 0.3$ & $-7.0 \pm 0.1$ \\
\hline $130948^{a}$. & 55.73 & 144.7 & 32.4 & -2.7 & $13.4 \pm 0.1$ & $21.0 \pm 0.2$ & $-0.6 \pm 0.1$ \\
\hline $141272^{\mathrm{a}} \ldots \ldots$ & 46.84 & -176.2 & -166.7 & -26.6 & $-10.1 \pm 0.1$ & $-16.2 \pm 0.5$ & $-6.4 \pm 0.1$ \\
\hline $203244 \ldots \ldots$ & 48.86 & 140.9 & 169.4 & 11.5 & $10.9 \pm 0.2$ & $20.5 \pm 0.3$ & $-15.5 \pm 0.3$ \\
\hline $217813 \ldots \ldots$ & 41.19 & -117.7 & -28.1 & 1.5 & $22.3 \pm 0.3$ & $15.5 \pm 0.1$ & $9.2 \pm 0.1$ \\
\hline
\end{tabular}

${ }^{\text {a }}$ Constant radial velocity.

b Possible spectroscopic binary.

${ }^{\mathrm{c}}$ Possible member of Ursa Major group.

season separately. We were able to derive photometric periods for 26 of the 31 stars that we observed with the APTs. These stars all have photometric amplitudes of roughly $0.05 \mathrm{mag}$ or less. The others had amplitudes too small for reliable period determinations. The photometric periods for the individual seasons were determined via standard periodogram techniques that are computatively equivalent to the method of Horne \& Baliunas (1986). Periods listed in column (5) are weighted means of the periods determined separately in the $B$ and $V$ bands (for the $0.40 \mathrm{~m} \mathrm{APT}$ ) or in the $b$ and $y$ bands (for the $0.75 \mathrm{~m} \mathrm{APT}$ ). The mean periods listed in column (6) are weighted means of the indi- vidual periods in column (5). Figure 1 shows the results of the periodogram analysis of HD 63433.

We interpret the photometric periods to be stellar rotation periods. Strong magnetic dynamos in these young, active stars produce sufficient star spot activity for rotational modulation of surface features to be evident in most light curves. Several stars show relatively unchanging rotation periods from year to year (HD 1835, 82443, 113449, and 152391); several others show a range of rotation periods (HD 20630, 30495, 97334, and 206860), which suggests a combination of differential rotation and concentration of star spot activity at different stellar latitudes from year to 
TABLE 2

Summary of Photometric Observations and Derived Periods

\begin{tabular}{|c|c|c|c|c|c|}
\hline $\begin{array}{c}\text { HD } \\
(1)\end{array}$ & $\begin{array}{c}\text { APT } \\
\text { (2) }\end{array}$ & $\begin{array}{c}\text { Date Range } \\
(\mathrm{JD}-2,400,000) \\
(3)\end{array}$ & $\begin{array}{c}N_{\text {obs }} \\
\text { (4) }\end{array}$ & $\begin{array}{l}\text { Period } \\
\text { (days) } \\
(5)\end{array}$ & $\begin{array}{c}\text { Mean Period } \\
\text { (days) } \\
(6)\end{array}$ \\
\hline $166 \ldots \ldots \ldots$ & 0.75 & $49,540-49,740$ & 33 & $6.23 \pm 0.01$ & 6.23 \\
\hline \multirow[t]{5}{*}{$1835 \ldots \ldots}$. & 0.75 & $49,239-49,356$ & 34 & $7.81 \pm 0.01$ & 7.81 \\
\hline & & $49,546-49,706$ & 29 & $7.77 \pm 0.04$ & \\
\hline & & $49,986-50,092$ & 27 & $7.68 \pm 0.09$ & \\
\hline & & $50,642-50,820$ & 33 & $7.77^{a} \pm 0.02$ & \\
\hline & & $51,085-51,197$ & 49 & $7.88 \pm 0.03$ & \\
\hline 7590 & 0.40 & $49,236-49,401$ & 76 & $5.67 \pm 0.01$ & 5.67 \\
\hline $10008 \ldots \ldots$ & 0.40 & $51,172-51,227$ & 34 & b & $\ldots$ \\
\hline \multirow[t]{2}{*}{$10780 \ldots \ldots$} & 0.75 & $49,239-49,389$ & 46 & $21.0 \pm 0.3$ & 21.7 \\
\hline & & $49,990-50,128$ & 63 & $22.5 \pm 0.4$ & \\
\hline \multirow{3}{*}{$11131 \ldots \ldots$} & 0.75 & $49,239-49,374$ & 56 & $8.77 \pm 0.04$ & 8.92 \\
\hline & & $49,624-49,724$ & 27 & $8.90 \pm 0.03$ & \\
\hline & & $49,986-50,101$ & 38 & $9.50 \pm 0.06$ & \\
\hline \multirow[t]{6}{*}{20630 . } & 0.75 & $29,239-49,412$ & 66 & $9.14 \pm 0.03$ & 9.20 \\
\hline & & $49,633-49,773$ & 42 & $9.14 \pm 0.05$ & \\
\hline & & $49,986-50,130$ & 59 & $9.21 \pm 0.04$ & \\
\hline & & $50,391-50,499$ & 31 & $9.15 \pm 0.03$ & \\
\hline & & $50,718-50,868$ & 34 & $9.10 \pm 0.04$ & \\
\hline & & $51,086-51,240$ & 52 & $9.46 \pm 0.03$ & \\
\hline $26923 .$. & 0.40 & $51,172-51,256$ & 48 & $\bar{b}$ & $\ldots$ \\
\hline \multirow[t]{6}{*}{$30495 \ldots \ldots}$. & 0.75 & $49,239-49,408$ & 68 & $11.47 \pm 0.08$ & 11.3 \\
\hline & & $49,633-49,786$ & 63 & $11.1 \pm 0.1$ & \\
\hline & & $49,986-50,123$ & 53 & $11.3 \pm 0.1$ & \\
\hline & & $50,393-50,510$ & 38 & $11.45 \pm 0.05$ & \\
\hline & & $50,718-50,877$ & 55 & $11.5 \pm 0.2$ & \\
\hline & & $51,087-51,238$ & 57 & $10.5 \pm 0.1$ & \\
\hline \multirow[t]{6}{*}{$37394 \ldots \ldots}$. & 0.75 & $49,239-49,449$ & 78 & $11.02 \pm 0.05$ & 10.86 \\
\hline & & $49,633-49,814$ & 61 & $10.78 \pm 0.05$ & \\
\hline & & $49,986-50,180$ & 74 & $10.86 \pm 0.03$ & \\
\hline & & $50,392-50,551$ & 51 & $10.84 \pm 0.04$ & \\
\hline & & $50,730-50,921$ & 81 & $\mathbf{b}$ & \\
\hline & & $51,087-51,283$ & 65 & b & \\
\hline 41593. & 0.40 & $51,170-51,287$ & 73 & $7.97 \pm 0.04$ & 7.97 \\
\hline $43162 \ldots \ldots$. & 0.40 & $51,170-51,261$ & 52 & $\overline{\mathrm{b}}$ & $\ldots$ \\
\hline $52698 \ldots \ldots$. & 0.40 & $51,170-51,266$ & 52 & b & $\ldots$ \\
\hline $63433 \ldots \ldots$ & 0.40 & $51,170-51,314$ & 109 & $6.46 \pm 0.01$ & 6.46 \\
\hline $72760 \ldots \ldots$ & 0.40 & $51,170-51,317$ & 115 & $\mathrm{~b}$ & $\ldots$ \\
\hline \multirow[t]{10}{*}{$72905^{\mathrm{c}} \ldots \ldots$} & 0.75 & $49,227-49,482$ & 91 & $4.62 \pm 0.01$ & 4.89 \\
\hline & & & & $5.28 \pm 0.01$ & \\
\hline & & $49,639-49,840$ & 86 & $4.68 \pm 0.01$ & \\
\hline & & & & $5.30 \pm 0.02$ & \\
\hline & & $50,013-50,214$ & 75 & $4.69 \pm 0.01$ & \\
\hline & & $50,405-50,577$ & 60 & $4.90 \pm 0.02$ & \\
\hline & & & & $5.46 \pm 0.02$ & \\
\hline & & $50,736-50,949$ & 83 & $4.68 \pm 0.01$ & \\
\hline & & & & $5.38 \pm 0.01$ & \\
\hline & & $51,096-51,317$ & 73 & $5.29 \pm 0.01$ & \\
\hline $73350 \ldots \ldots$ & 0.40 & $51,172-51,316$ & 101 & $6.14 \pm 0.02$ & 6.14 \\
\hline \multirow{7}{*}{$82443 \ldots \ldots .}$. & 0.40 & $48,906-49,146$ & 111 & $5.45 \pm 0.01$ & 5.42 \\
\hline & & $49,272-49,511$ & 165 & $5.52 \pm 0.01$ & \\
\hline & & $49,638-49,878$ & 106 & $5.40 \pm 0.01$ & \\
\hline & & $49,996-50,244$ & 148 & $5.42 \pm 0.01$ & \\
\hline & & $50,392-50,608$ & 145 & $5.44 \pm 0.01$ & \\
\hline & & $50,728-50,976$ & 167 & $5.39 \pm 0.01$ & \\
\hline & & $51,094-51,342$ & 197 & $5.43 \pm 0.01$ & \\
\hline \multirow[t]{7}{*}{$97334 \ldots \ldots .}$. & 0.75 & $49,095-49,161$ & 41 & $8.3 \pm 0.1$ & 8.25 \\
\hline & & $49,312-49,519$ & 99 & $\bar{b}$ & \\
\hline & & $49,687-49,891$ & 72 & $8.56 \pm 0.04$ & \\
\hline & & $50,052-50,252$ & 86 & $\bar{b}$ & \\
\hline & & $50,404-50,629$ & 57 & $8.11^{\mathrm{a}} \pm 0.03$ & \\
\hline & & $50,768-50,991$ & 80 & $8.21 \pm 0.05$ & \\
\hline & & $51,123-51,334$ & 53 & $8.29 \pm 0.03$ & \\
\hline \multirow[t]{2}{*}{$109011 \ldots \ldots$} & 0.40 & $51,170-51,242$ & 67 & $8.56 \pm 0.02$ & 8.81 \\
\hline & & $51,291-51,360$ & 52 & $9.19 \pm 0.03$ & \\
\hline
\end{tabular}


TABLE 2-Continued

\begin{tabular}{|c|c|c|c|c|c|}
\hline $\begin{array}{l}\text { HD } \\
(1)\end{array}$ & $\begin{array}{l}\text { APT } \\
(2)\end{array}$ & $\begin{array}{c}\text { Date Range } \\
(\mathrm{JD}-2,400,000) \\
(3)\end{array}$ & $\begin{array}{c}N_{\text {obs }} \\
\text { (4) }\end{array}$ & $\begin{array}{l}\text { Period } \\
\text { (days) } \\
(5)\end{array}$ & $\begin{array}{l}\text { Mean Period } \\
\text { (days) } \\
\text { (6) }\end{array}$ \\
\hline \multirow[t]{6}{*}{$113449 \ldots \ldots$} & \multirow[t]{6}{*}{0.40} & $49,321-49,547$ & 124 & b & \multirow[t]{6}{*}{6.54} \\
\hline & & $49,701-49,908$ & 104 & $6.53 \pm 0.02$ & \\
\hline & & $50,053-50,261$ & 128 & $6.53 \pm 0.01$ & \\
\hline & & $50,419-50,635$ & 105 & $6.53 \pm 0.01$ & \\
\hline & & $50,788-50,993$ & 110 & $6.56 \pm 0.01$ & \\
\hline & & $51,153-51,360$ & 106 & $\bar{b}$ & \\
\hline $116956 \ldots \ldots$ & 0.40 & $51,171-51,360$ & 151 & $7.80 \pm 0.02$ & 7.80 \\
\hline $128987 \ldots \ldots$ & 0.40 & $51,181-51,360$ & 114 & $9.35 \pm 0.04$ & 9.35 \\
\hline $130948^{\mathrm{d}} \ldots \ldots$ & 0.40 & $51,172-51,360$ & 139 & $\begin{array}{l}7.69 \pm 0.03 \\
7.99 \pm 0.03\end{array}$ & 7.85 \\
\hline $135599 \ldots \ldots$ & 0.40 & $51,177-51,360$ & 112 & $5.97 \pm 0.02$ & 5.97 \\
\hline $141272 \ldots \ldots$ & 0.40 & $51,183-51,360$ & 114 & $14.01 \pm 0.04$ & 14.01 \\
\hline \multirow[t]{7}{*}{$152391 \ldots \ldots$} & \multirow[t]{7}{*}{0.75} & $49,095-49,163$ & 31 & $11.5 \pm 0.1$ & \multirow[t]{7}{*}{11.5} \\
\hline & & $49,399-49,547$ & 36 & $\bar{b}$ & \\
\hline & & $49,755-49,904$ & 29 & $11.4 \pm 0.1$ & \\
\hline & & $50,161-50,257$ & 31 & $11.5 \pm 0.1$ & \\
\hline & & $50,490-50,642$ & 41 & $11.4 \pm 0.1$ & \\
\hline & & $50,857-50,995$ & 45 & $11.5 \pm 0.1$ & \\
\hline & & $51,223-51,341$ & 40 & $\overline{\mathrm{b}}$ & \\
\hline $180161 \ldots \ldots$ & 0.40 & $51,233-51,359$ & 74 & $9.7 \pm 0.1$ & 9.7 \\
\hline \multirow[t]{6}{*}{$206860 \ldots \ldots$} & \multirow[t]{6}{*}{0.75} & $49,126-49,337$ & 65 & $4.73 \pm 0.01$ & \multirow[t]{6}{*}{4.91} \\
\hline & & $49,491-49,706$ & 54 & $4.64 \pm 0.01$ & \\
\hline & & $49,862-50,071$ & 43 & $5.10 \pm 0.01$ & \\
\hline & & $50,392-50,443$ & 20 & $\mathrm{e}$ & \\
\hline & & $50,620-50,806$ & 37 & $5.18 \pm 0.01$ & \\
\hline & & $51,077-51,179$ & 48 & $4.68 \pm 0.04$ & \\
\hline \multirow[t]{3}{*}{$217813 \ldots \ldots$} & \multirow[t]{3}{*}{0.75} & $49,145-49,362$ & 51 & $\frac{1}{b}$ & \multirow[t]{3}{*}{8.05} \\
\hline & & $49,512-49,666$ & 30 & $8.04 \pm 0.01$ & \\
\hline & & $49,879-50,091$ & 57 & $8.10 \pm 0.02$ & \\
\hline $220182 \ldots \ldots$ & 0.40 & $51,172-51,360$ & 48 & $7.66 \pm 0.01$ & 7.66 \\
\hline
\end{tabular}

${ }^{a}$ Period and its error have been multiplied by two.

b Photometric variability too small to determine period.

${ }^{c}$ Both the 4.7 and 5.3 day-periods often appear in the same observing season.

d Both periods appear in the same observing season.

e Insufficient data to determine period.

year. Two stars (HD 72905 and HD 130948) show two prominent rotation periods in the same observing season.

New and previously reported spectroscopic measurements appear in Table 3. Luminosities, L, are from Hipparcos parallax and visual magnitude measurements (Perryman et al. 1997) and an empirical bolometric correction (Flower 1996). Effective temperatures, $T_{\text {eff }}$, were estimated from the relation of Flower (1996). The emission in the $\mathrm{Ca}$ II $\mathrm{H}$ and $\mathrm{K}$ lines, a well-established indicator of stellar chromospheric activity, is expressed in terms of the Mount Wilson index,

$$
S_{\mathrm{MW}}=12 a \frac{C_{\mathrm{K}}+C_{\mathrm{KH}}}{C_{V}+C_{R}},
$$

where $C_{\mathrm{K}}$ and $C_{\mathrm{KH}}$ are the total measured signal in two triangular passbands, each with FWHM of $1.09 \AA$ and centered on the $\mathrm{K}$ and $\mathrm{H}$ line cores, and $C_{V}$ and $C_{R}$ are the measured signal in $20 \AA$-wide regions of the continuum blueward and redward of the lines (Vaughan, Preston, \& Wilson 1978). The constant $a \approx 0.76$, which incorporates spectral response differences between the KPNO coudé echelle and the original Mount Wilson spectrophotometer, was obtained by comparing our measurements with known $S$ values for three sample stars (HD 1835, 30495, and 72905) listed in Table 3 of Duncan et al. (1991). The index $R_{\mathrm{HK}}^{\prime}$, the logarithmic flux ratio of $\mathrm{Ca}$ II in emission to the bolometric flux of the photosphere, was calculated from the $S_{\mathrm{Mw}}$ values of each star (Noyes et al. 1984). Calibration errors are created by the long-term variability in the $S$ index, $0.02-$ 0.03 for the calibration stars (Radick et al. 1998; Wilson 1978). We estimate our $R_{\mathrm{HK}}^{\prime}$ values have an absolute uncertainty of $0.03-0.05$ due to calibration errors alone. Thus while our measurements are not appropriate for sensitive estimates of variability, they are sufficient to distinguish clearly active from relatively inactive (Sun-like) stars. Values of this index and revised values of the logarithmic index of coronal X-ray flux relative to bolometric flux $R_{\mathrm{X}}$ from Gaidos (1998) are reported in Table 3.

Spectroscopy in the vicinity of the $6707.8 \AA$ line of Li I was obtained for 34 stars and is reported in Table 3 . The equivalent widths of the blended Li I lines in the vicinity of $6707.8 \AA$ were obtained by least-squares fitting of a model that included the Li I doublet at 6707.761 and $6707.912 \AA$, two $\mathrm{Fe}$ I doublets at 6703.568 and $6705.101 \AA$, and two $\mathrm{Fe}$ I lines at 6707.441 and $6710.316 \AA$. The strength of each of the iron lines was independently fitted except for the line at $6707.441 \AA$, which is often blended with the Li I lines in the spectra of these rapidly rotating stars. Thus we fixed the intensity of this line relative to one of the Fe I lines, estimating the scaling factor from spectra of lithium-depleted old stars. This scaling factor is not constant but varies with the 

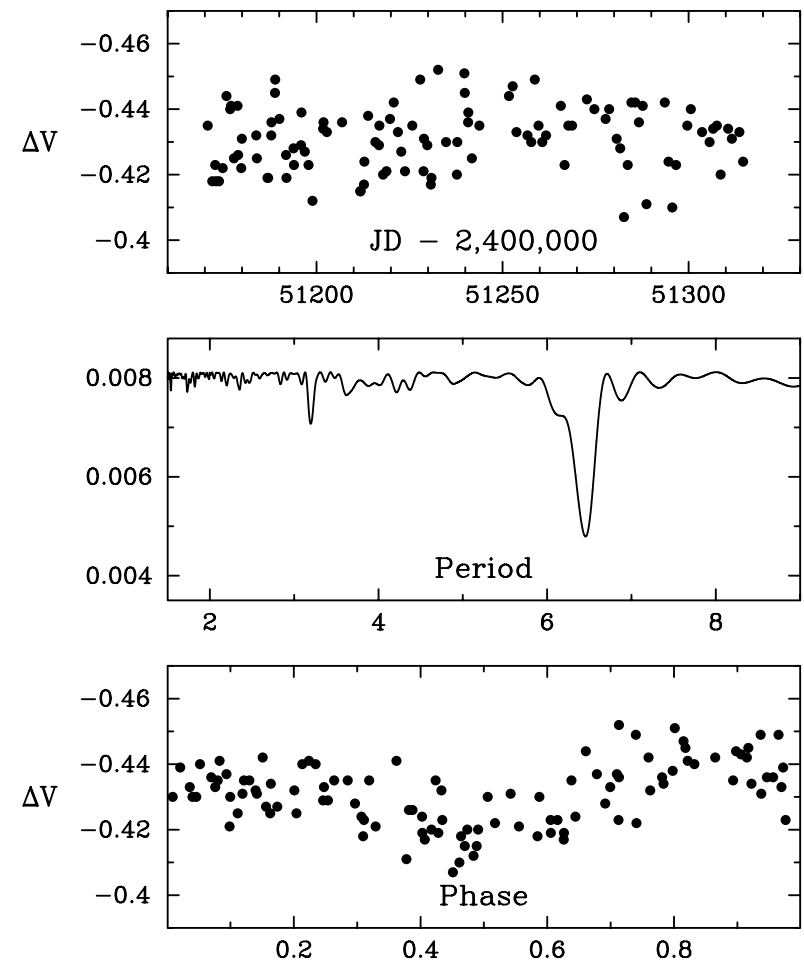

FIG. 1.-Top: Johnson $V$ photometric observations of HD 63443 plotted against the Julian Date. Middle: Periodogram of the HD 63443 observations. The sum of the squares of the residuals from a least-squares sine fit are plotted against the trial period in days. The resulting period is $6.46 \pm 0.01$ days. Bottom: The photometric observations from the top panel plotted against the photometric phase computed with the 6.46 day period and the arbitrary epoch JD 2,451,170.

stellar $T_{\text {eff }}$. However, this effect is important only when Li I lines are very weak. A correction of $3.5 \%$ was applied to the equivalent widths to account for scattered light (J. Cardelli 1994, unpublished). Errors in the equivalent width of the lithium line are typically $\sim 1 \mathrm{~m} \AA$. For consistency, abundance estimates with respect to hydrogen were estimated from interpolations of the curve of growth published by Soderblom et al. (1993). Lithium abundances relative to hydrogen $(\log N=12)$ and values obtained by Pasquini, Liu, \& Pallavicini (1994) for two southern stars not observed by us are given as well.

The projected rotational velocity $(v \sin i)$ of each star A was estimated by cross-correlating its spectrum with that of a more slowly rotating template star $\mathrm{B}$. The additional broadening due to the more rapid rotation of $\mathrm{A}$ is the difference in quadrature between the width of the correlation peak $\sigma_{\mathrm{AB}}$ and the width of the autocorrelation peak $\sigma_{\mathrm{BB}}$. Differencing the widths of the correlation and autocorrelation spectra removes the contribution of intrinsic line widths and instrument broadening. The $v \sin i$ value is calculated by adding the rotational velocity of the template in quadrature to the result,

$$
(v \sin i)_{\mathrm{A}}=\sqrt{\sigma_{\mathrm{AB}}^{2}-\sigma_{\mathrm{BB}}^{2}+(v \sin i)_{\mathrm{B}}^{2}} .
$$

Estimates for stars with multiple measurements are averaged. The mean measurement error in $v \sin i$ is $0.28 \mathrm{~km}$ but the rms differences between multiple measurements averaged over all stars is $0.63 \mathrm{~km} \mathrm{~s}^{-1}$. The observed inclination of the star with respect to the line-of-sight ( 0 indicates poleon) was computed from our measured $v \sin i$ values and rotation periods, along with each star's radius estimated from its luminosity and $T_{\text {eff }}$. The range of possible inclinations for each star listed in Table 3 reflects the uncertainties in all of these parameters. Most of the uncertainty comes from the $v \sin i$ measurements. We included an additional error of $0.5 \mathrm{~km} \mathrm{~s}^{-1}$ in $v \sin i$ to account for uncertainties in the microturbulence parameter.

\section{DISCUSSION}

Radial velocity measurements now rule out close $(a<1$ AU) stellar-mass companions with $\sin i>20^{\circ}$ around 16 stars (Table 1). One star (HD 113449) may be a spectroscopic binary, exhibiting a change of $20 \mathrm{~km} \mathrm{~s}^{-1}$ in 10 months. Measurements using low-resolution $\left(3.6 \mathrm{~km} \mathrm{~s}^{-1}\right.$ accuracy) prism spectra obtained at two epochs also suggest velocity variation (Moore \& Paddock 1950), but additional confirming observations are needed. If this star is a spectroscopic binary, by the criteria of Gaidos (1998) this system should not be considered a solar analog. We confirm the constant and anomalously high motions of HD 152391 and HD 220182 (137 and $59 \mathrm{~km} \mathrm{~s}^{-1}$, respectively). The radial velocity of a third star (HD 73350) was erroneously reported (Gaidos 1998), and although its correct space motion is high $\left(35 \mathrm{~km} \mathrm{~s}^{-1}\right)$, it is not anomalous.

The remaining 34 stars with known space motions are plotted in Figure 2. Integrals of the motion reflect the ancestral dynamics of a star and any subsequent perturbations by close encounters with other stars and molecular clouds. Within the restricted ( $25 \mathrm{pc}$ radius) volume of the sample space, velocities are quantitative proxies for the three integrals of motion describing nearly circular orbits in the axisymmetric gravitational potential of the Galactic disk, i.e., $E_{z}=1 / 2\left(V^{2}+\kappa_{0}^{2} y^{2}\right), E_{z}=1 / 2\left(W^{2}+v_{0}^{2} z^{2}\right)$, and $L=U$ $+y \Omega_{0}$, where $y$ and $z$ are the right-handed Galactic coordinate distances from the Sun, $\kappa_{0}$ and $v_{0}$ are the epicycle frequencies evaluated at the LSR, and $\Omega_{0}$ is the angular velocity of the LSR (Binney \& Tremaine 1987). Since the coefficients $\kappa_{0}, v_{0}$, and $\Omega_{0}$ are small, with estimated values of $0.036,0.100$, and $0.014 \mathrm{~km} \mathrm{~s}^{-1} \mathrm{pc}^{-1}$, respectively, the spatial terms in the integrals of motion can be neglected compared to the velocity terms. In the following discussion, we consider these stars according to the their clustering into common-motion groups.

The short rotation periods and high Ca II HK emission of these objects relative to the Sun (Fig. 3) are consistent with their selection as active stars with exceptionally X-ray luminous coronae, with one exception (discussed below). The $\mathrm{X}$-ray emission of these stars exhibits considerable scatter from the empirical relation with rotation $L_{\mathrm{X}} \sim P^{-2.46}$ formulated by Güdel, Guinan, \& Skinner (1997; solid line). $\mathrm{X}$-ray and $\mathrm{Ca}$ II emission are also not strongly correlated. This may in part be due to variability in activity and the 8-9 $\mathrm{yr}$ that elapsed between the X-ray measurements and the Ca II HK measurements. The photospheric lithium abundances are plotted in Figure 4, where the stars are identified according to their space motion group affiliation, if any. The Li abundance of stars in the Pleiades (65 Myr; Soderblom \& Mayor 1993) and Hyades (650 Myr; Perryman et al. 1998) dwarfs are shown for comparison. The values are typical of stars with ages within this range and follow the well-established inverse trend with $T_{\text {eff }}$.

Seven young solar analogs (Table 3 ) move within $5 \mathrm{~km}$ $\mathrm{s}^{-1}$ of a mean motion consistent with the previously described Ursa Major kinematic group (Soderblom \& 
TABLE 3

ACTIVITY, LITHIUM, AND ROTATION

\begin{tabular}{|c|c|c|c|c|c|c|c|c|}
\hline HD & $\begin{array}{c}L \\
\left(L_{\odot}\right)\end{array}$ & $\begin{array}{l}T_{\text {eff }} \\
(\mathrm{K})\end{array}$ & $R_{\mathrm{X}}$ & $R_{\mathrm{HK}}^{\prime}$ & $\begin{array}{l}W_{6708} \\
(\mathrm{~m} \AA)\end{array}$ & $\begin{array}{c}\log N(\mathrm{Li}) \\
(\mathrm{H}=12)\end{array}$ & $\begin{array}{c}v \sin i \\
\left(\mathrm{~km} \mathrm{~s}^{-1}\right)\end{array}$ & $\begin{array}{c}i \\
(\mathrm{deg})\end{array}$ \\
\hline \multicolumn{9}{|c|}{ Ursa Major group: } \\
\hline $26923 \ldots \ldots$ & 1.10 & 6010 & -4.64 & -4.52 & $79.2 \pm 3.4$ & $2.73 \pm 0.09$ & 4.2 & \\
\hline $41593 \ldots \ldots$ & 0.45 & 5360 & -4.59 & -4.36 & $12.8 \pm 3.5$ & $1.22 \pm 0.17$ & 5.0 & $50-90$ \\
\hline $63433 \ldots \ldots$ & 0.73 & 5580 & -4.55 & -4.34 & $78.9 \pm 2.3$ & $2.33 \pm 0.10$ & 6.1 & $40-90$ \\
\hline $72905 \ldots \ldots$ & 0.97 & 5820 & -4.47 & -4.42 & $108.5 \pm 5.0$ & $2.80 \pm 0.10$ & 8.7 & $45-90$ \\
\hline $109011 \ldots \ldots$ & 0.35 & 4760 & -4.74 & -4.36 & $24.7 \pm 3.4$ & $0.81 \pm 0.15$ & 5.5 & $55-90$ \\
\hline $135599 \ldots \ldots$ & 0.40 & 5080 & -4.94 & $\ldots$ & $3.7 \pm 1.9$ & $0.34 \pm 0.28$ & 4.6 & $30-65$ \\
\hline $165185 \ldots \ldots$ & 1.08 & 5810 & -4.51 & -4.49 & $92.7 \pm 3.3$ & $2.65 \pm 0.10$ & 7.2 & $\ldots$ \\
\hline \multicolumn{9}{|c|}{ Local association: } \\
\hline $166 \ldots \ldots \ldots$ & 0.65 & 5310 & -4.38 & -4.38 & $71.2 \pm 0.5$ & $1.98 \pm 0.11$ & 4.1 & $20-45$ \\
\hline $37394 \ldots \ldots$ & 0.48 & 5200 & -4.74 & -4.44 & $1.3 \pm 3.2$ & $0.03 \pm 0.03$ & 4.0 & $45-90$ \\
\hline $97334 \ldots \ldots \ldots$ & 1.08 & 5890 & -4.57 & -4.41 & $81.1 \pm 2.8$ & $2.64 \pm 0.09$ & 5.6 & $45-90$ \\
\hline $116956 \ldots \ldots$ & 0.55 & 5170 & -4.48 & $\ldots$ & $31.0 \pm 2.6$ & $1.42 \pm 0.12$ & 5.6 & $45-90$ \\
\hline $206860 \ldots \ldots$ & 1.17 & 5930 & -4.46 & -4.41 & $100.8 \pm 3.7$ & $2.81 \pm 0.09$ & 9.4 & $45-90$ \\
\hline \multicolumn{9}{|c|}{ Possible Pleiades cluster: } \\
\hline $82443 \ldots \ldots$ & 0.45 & 5240 & -4.01 & -4.22 & $183.9 \pm 2.8$ & $2.61 \pm 0.12$ & 6.1 & $35-90$ \\
\hline $113449 \ldots \ldots$ & 0.41 & 5020 & -4.41 & $\ldots$ & $139.8 \pm 2.7$ & $2.08 \pm 0.12$ & 5.8 & $40-90$ \\
\hline \multicolumn{9}{|c|}{ Possible Hyades cluster: } \\
\hline $73350 \ldots \ldots$ & 0.97 & 5750 & -4.85 & -4.49 & $36.0 \pm 3.5$ & $2.09 \pm 0.10$ & 3.8 & $20-40$ \\
\hline $180161 \ldots \ldots$ & 0.58 & 5170 & -4.67 & -4.44 & $9.3 \pm 1.6$ & $0.86 \pm 0.14$ & 3.1 & $25-60$ \\
\hline \multicolumn{9}{|c|}{ High-velocity stars: } \\
\hline $152391 \ldots \ldots$ & 0.58 & 5230 & -4.63 & -4.44 & $6.4 \pm 2.4$ & $0.77 \pm 0.21$ & 3.7 & $40-90$ \\
\hline $220182 \ldots \ldots$ & 0.52 & 5150 & -4.59 & -4.34 & $47.3 \pm 3.6$ & $1.59 \pm 0.12$ & 5.0 & $40-90$ \\
\hline \multicolumn{9}{|l|}{ Old star: } \\
\hline $10780 \ldots \ldots$ & 0.53 & 5420 & -4.89 & -4.71 & $1.3 \pm 2.21$ & $0.27 \pm 0.27$ & 2.0 & $35-90$ \\
\hline \multicolumn{9}{|l|}{ Others: } \\
\hline $1237 \ldots \ldots \ldots$ & 0.66 & 5340 & -4.46 & -4.44 & $\ldots$ & . & 0.0 & $\ldots$ \\
\hline $1835 \ldots \ldots \ldots$ & 1.00 & 5860 & -4.55 & -4.46 & $76.7 \pm 4.1$ & $2.58 \pm 0.10$ & $\ldots$ & $\ldots$ \\
\hline $7590 \ldots \ldots \ldots$ & 1.08 & 5930 & -4.72 & -4.46 & $92.1 \pm 4.3$ & $2.75 \pm 0.10$ & 6.4 & $35-65$ \\
\hline $10008 \ldots \ldots$ & 0.46 & 5170 & -4.48 & -4.38 & $88.7 \pm 2.3$ & $1.96 \pm 0.12$ & 2.9 & $\ldots$ \\
\hline $11131 \ldots \ldots$ & 0.92 & 5790 & -4.37 & -4.47 & $64.5 \pm 3.3$ & $2.42 \pm 0.10$ & $\ldots$ & $\ldots$ \\
\hline $20630 \ldots \ldots$ & 0.85 & 5660 & -4.65 & -4.44 & $35.0 \pm 3.6$ & $2.00 \pm 0.11$ & 4.5 & $40-90$ \\
\hline $30495 \ldots \ldots$ & 0.97 & 5830 & -4.77 & -4.56 & $47.0 \pm 3.5$ & $2.29 \pm 0.10$ & 4.1 & $45-90$ \\
\hline $36435 \ldots \ldots$ & 0.56 & 5460 & -4.74 & -4.44 & $23.0 \pm 5.0$ & $1.60 \pm 0.15$ & $\ldots$ & $\ldots$ \\
\hline $43162 \ldots \ldots$ & 0.71 & 5480 & -4.31 & -4.40 & $60.3 \pm 2.7$ & $2.07 \pm 0.11$ & 5.7 & $\ldots$ \\
\hline $52698 \ldots \ldots$ & 0.45 & 4930 & -4.75 & -4.59 & $0.2 \pm 3.0$ & $\ldots$ & 3.5 & $\ldots$ \\
\hline $59967 \ldots \ldots$. & 0.88 & 5730 & -4.48 & -4.44 & $81.4 \pm 3.6$ & $2.50 \pm 0.10$ & 3.4 & $\ldots$ \\
\hline $72760 \ldots \ldots$ & 0.53 & 5210 & -4.78 & -4.39 & $4.4 \pm 3.3$ & $0.57 \pm 0.45$ & 4.2 & $\ldots$ \\
\hline $118972 \ldots \ldots$ & 0.42 & 4990 & -4.67 & -4.40 & $3.7 \pm 3.4$ & $0.24 \pm 0.29$ & 5.2 & $\ldots$ \\
\hline $128400 \ldots \ldots$ & 0.75 & 5480 & -4.83 & -4.56 & $\ldots$ & $\ldots$ & $\ldots$ & $\ldots$ \\
\hline $128987 \ldots \ldots$ & 0.63 & 5590 & -4.78 & $\ldots$ & $25.8 \pm 2.3$ & $1.78 \pm 0.11$ & 3.2 & $30-80$ \\
\hline $130948 \ldots \ldots$ & 1.21 & 5730 & -4.69 & -4.45 & $98.4 \pm 2.4$ & $2.61 \pm 0.10$ & 6.0 & $40-90$ \\
\hline $141272 \ldots \ldots$ & 0.46 & 5170 & -4.62 & $\ldots$ & $3.9 \pm 1.9$ & $0.48 \pm 0.27$ & 4.0 & $70-90$ \\
\hline $203244 \ldots \ldots$ & 0.61 & 5530 & -4.61 & -4.39 & $22.0 \pm 5.0$ & $1.64 \pm 0.15$ & $\ldots$ & $\ldots$ \\
\hline $217813 \ldots \ldots$ & 1.09 & 5810 & -4.55 & -4.47 & $78.0 \pm 3.4$ & $2.54 \pm 0.10$ & 3.1 & $20-40$ \\
\hline
\end{tabular}

Mayor 1993) at $(U, V, W)=(+2.4,+12.2,-3.3) \mathrm{km} \mathrm{s}^{-1}$ (Fig. 2). The age of the Ursa Major comoving group has been estimated as $300 \mathrm{Myr}$, based on several techniques (Giannuzzi 1979). The lithium abundances of the seven members are intermediate between those of the Pleiades (70 Myr) and Hyades (625 Myr) dwarfs, and thus consistent with the estimated age. Five stars have motions within $3 \mathrm{~km}$ $\mathrm{s}^{-1}$ of a mean of $(-6.1,-10.1,-3.7) \mathrm{km} \mathrm{s}^{-1}$, consistent with previous descriptions of a local association (Jeffries 1995). A link between the Local Association and the Pleiades Cluster has been proposed (Eggen 1998 and references therein). We estimate a mean space motion of the Pleiades cluster of $(+8.6,-1.0,+1.4)$ based on a mean radial velocity of $5.85 \mathrm{~km} \mathrm{~s}^{-1}$ and Hipparcos proper motions of 17 of its brightest members (Eggen 1998). This motion is significantly different $\left(\Delta U=8.8 \mathrm{~km} \mathrm{~s}^{-1}\right)$ from that of the Local Association, and we continue to believe that these two groups are kinematically distinct entities. Furthermore, none of the five Local Association stars in this sample have the characteristics expected of Pleiades-age, solar-mass stars, i.e., $\log L_{\mathrm{X}} \sim(-3.5)$ and $\log N(\mathrm{Li}) \sim 3$. Rather, our data suggest that the Local Association is a group of stars similar in age to the Ursa Major group. However, the TW Hydra association, a group of low-mass stars that includes the 10 to $20 \mathrm{Myr}-$ old T Tauri archetype (Kastner et al. 1997; Webb et al. 1999; Sterzik et al. 1999) appears to move at $(-4.3,-9.4,-2.5) \mathrm{km} \mathrm{s}^{-1}$, i.e., within about $2 \mathrm{~km} \mathrm{~s}^{-1}$ of the Local Association. More accurate proper-motion measurements of TW Hya stars are needed. A common motion would indicate either a common origin of the two groups (although not a common age) or suggest that young stars in the solar neighborhood tend to form 

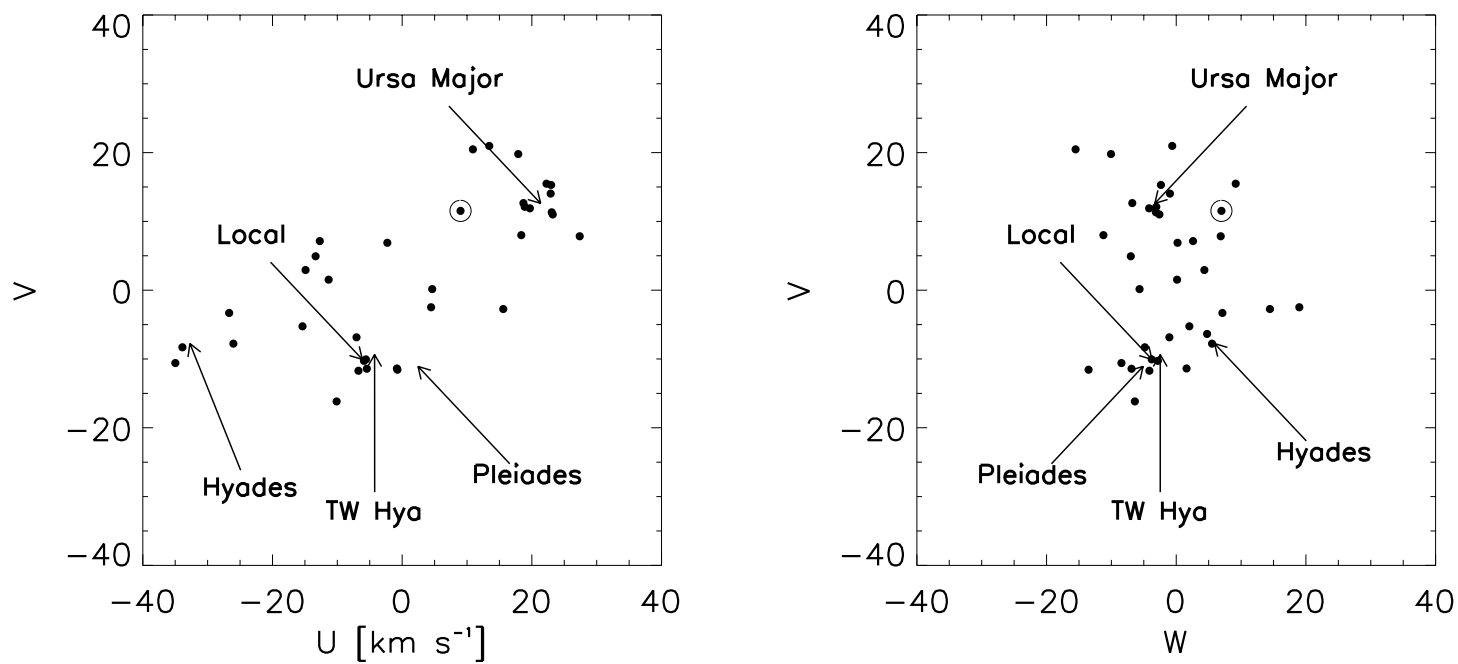

FIG. 2.-Space motions of 34 of the 38 young solar analogs with respect to the LSR. Two stars in the sample (HD 1237 and HD 128400 ) lack radial velocity information, and two others (HD 152391 and HD 220182) have anomalously high space motions that fall outside the plot. The Sun is shown as the encircled point. The motions of the Ursa Major, Local Association, and TW Hydra kinematic groups and the Pleiades and Hyades clusters are labeled.

with the same motion, perhaps that of a local molecular cloud component of the disk (the Ursa Major Group being a clear exception).

HD 82443 (DX Leo) is an exceptionally active star in our survey that moves within $7.4 \mathrm{~km} \mathrm{~s}^{-1}$ of the Pleiades and 5.7 $\mathrm{km} \mathrm{s}^{-1}$ of the TW Hydra association. The common motion of HD 82443 and the Pleiades was pointed out by Soderblom \& Clements (1988), and the observed lithium abundance, rapid rotation, high Ca II HK emission, and high X-ray luminosity (by far the highest in the sample) support an exceptionally young age. Its apparent rotation period was observed to vary from 5.39 to 5.52 days, consistent with

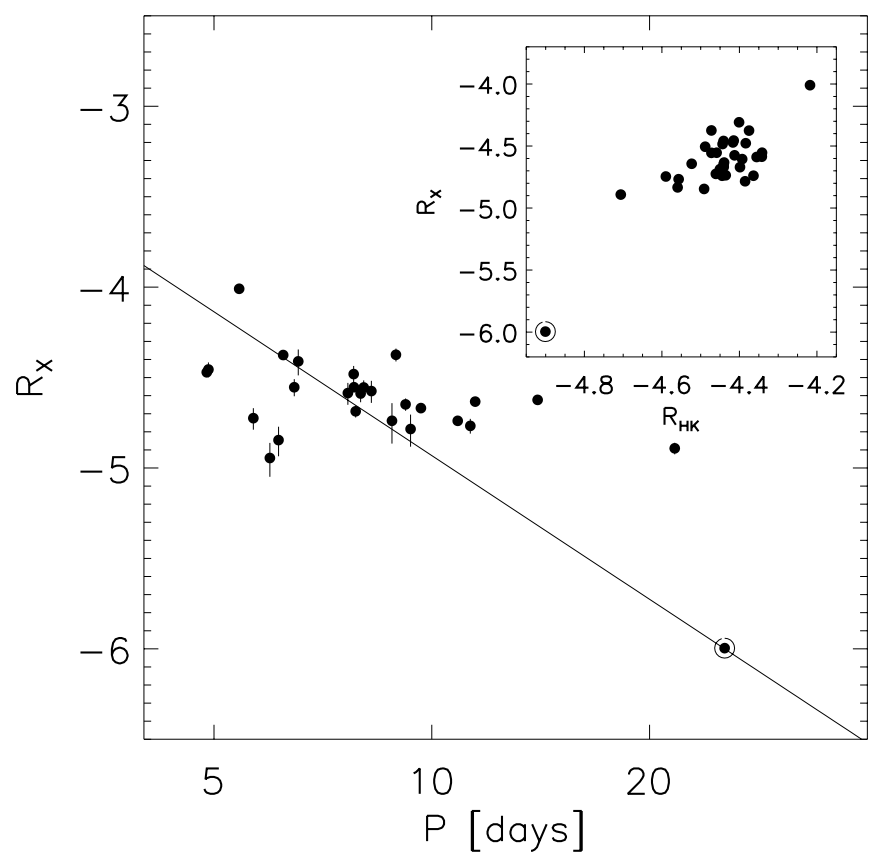

FIG. 3.-Index of coronal X-ray emission $R_{\mathrm{X}}$ plotted vs. rotation period for young solar analogs. The solid line represents the empirical relation from Güdel, Guinan, \& Skinner (1997). The inset shows the correlation between X-ray emission and the equivalent index for emission in the Ca II $\mathrm{K}$ and $\mathrm{H}$ lines. The encircled point is the Sun. a previous estimate (Messina et al. 1999). We derive a value of $v \sin i$ of $6.1 \pm 0.2 \mathrm{~km} \mathrm{~s}^{-1}$, in agreement with Benz \& Mayor (1981). The $(U, V)$-space motions of HD 113449 also lie within the range $3-4 \mathrm{~km} \mathrm{~s}^{-1}$ of the Pleiades and the Local Association. This could be merely a coincidence if the system is a single-lined spectroscopic binary; however, the high lithium abundance suggests a Pleiades age as well. Stars in the Hyades cluster, 46 pc distant, have been identified as far as $20 \mathrm{pc}$ from the cluster center (Perryman et al. 1998), and thus members of this $\sim 625 \mathrm{Myr}$-old cluster may appear in our sample. Two stars, HD 73350 and HD 180161, have $(U, V)$-space motions within $4 \mathrm{~km} \mathrm{~s}^{-1}$ of the mean cluster motion of $(-32.7,-7.8,+5.9)$ with respect to the LSR, although the $W$-components of their motion differ by $10-15 \mathrm{~km} \mathrm{~s}^{-1}$. Their rotation, activity and lithium abundances suggest a Hyades-like age.

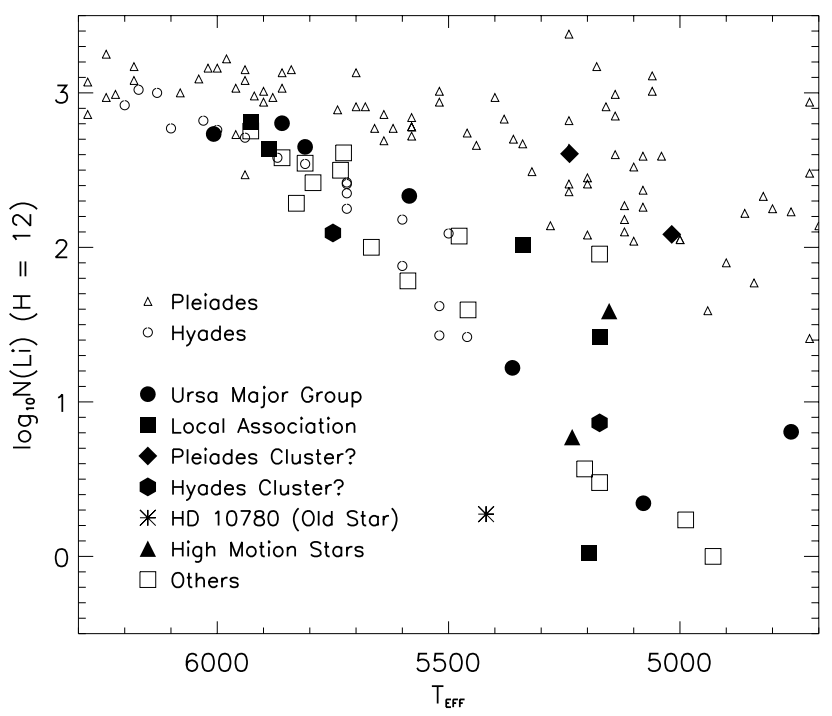

Fig. 4.- Lithium abundances relative to hydrogen for 34 solar analogs estimated from the strength of the Li I doublet at $6707.8 \AA$. Dwarf stars in the $70 \mathrm{Myr}$-old Pleiades (Soderblom et al. 1993) and $625 \mathrm{Myr}-$ old Hyades (Soderblom et al. 1990) clusters are shown for comparison. 
The K0 star HD 10780 has a rotation period of 21.67 days, much longer than the other stars in the sample, indicative of a solar age. For example, Lachaume et al. (1999) assigned an age of 2.2-3.5 Gyr, based on a previous measurement of 23 days (Noyes et al. 1984). A long rotation period, combined with a nearly pole-on-viewing geometry, would explain the very low $v$ sin $i$ value $0.6 \mathrm{~km} \mathrm{~s}^{-1}$ (Fekel 1997). From an estimate of the FWHM of $0.10 \AA$ from our new spectra, the total broadening in the stellar spectrum is 2 $\mathrm{km} \mathrm{s}^{-1}$. This is roughly equal to an estimate of the macroturbulence parameter (Saar \& Osten 1997). Thus the actual value of $v \sin i$ for HD 10780 could be even smaller. The star is not a member of any of the identified kinematic groups. Its lithium abundance is roughly solar, also suggesting an old age. However, the observed $\mathrm{Ca}$ II emission index $S=0.26$ (cf. the value of 0.32 found by Duncan et al. in 1979) exceeds the solar maximum value by 10 times the normal solar variation (Radick et al. 1998). Likewise, HD 10780 is 10 times more X-ray luminous than the Sun at solar maximum $\left(R_{\mathrm{X}} \approx-5.8\right)$. As the optical and $\mathrm{X}$-ray data were obtained at different epochs, the different observations cannot be explained by a single flare event. Radial velocity data rule out stellar companions on nearly circular orbits closer than 10 AU. A spectrum of SAO 11985, a putative $m_{V}=9.69$ companion $48^{\prime \prime}$ from HD 10780, reveals that it is a more distant giant star with line-of-sight velocity $36 \mathrm{~km}$ $\mathrm{s}^{-1}$ relative to HD 10780 and is unlikely to be the source of X-ray emission. Regardless, a companion could not explain the Ca II HK emission. Since there is no evidence that the Sun's activity is anomalous with respect to other solar-mass stars of its age (Soderblom 1983), we conclude that HD 10780 is a relatively active star of solar age and not a young analog. In fact, the reestimated value of $R_{\mathrm{X}}$ falls just below the original selection cutoff of Gaidos (1998).

An explanation for the nature of the two high-velocity stars (HD 152391 and HD 220182) in our sample remains elusive. Their space motions and their location well above the zero-age main sequence suggest that these are evolved stars from an old disk or halo population. Radial velocity measurements at multiple epochs (ours and those in the literature) rule out the possibility that either of these objects are spectroscopic binaries. Moreover, the high Ca II HK emission, short rotation periods, and high $v \sin i$ values unambiguously confirm that each star (and not some unknown companion) is active. Even more enigmatic is the high abundance of lithium in HD $220182(\log N(\mathrm{Li}) \approx 1.6)$, characteristic of an age of 200-300 Myr rather than $\sim 10$ Gyr. It would appear that the convecting outer layers of these stars have recently acquired a source of angular momentum, and in one case, lithium. Simon \& Drake (1989) proposed that the rapid rotation and high chromosopheric activity seen in a small fraction of $\mathrm{G}$ and $\mathrm{K}$ giant and subgiant stars is the result of convective dredge-up of material from a rapidly rotating core as the stars evolve to the base of the red giant branch. The high lithium abundance seen in asymptotic giant branch stars has been explained by lithium production via decay of ${ }^{7} \mathrm{Be}$ (Cameron \& Fowler 1971) and rapid convective transport to the surface (Fekel \& Balachandran 1993), but this mechanism may not be responsible for all cases (King, Deliyannis, \& Boesgaard 1996). It is not clear whether the required temperatures are reached in evolved, low-mass stars. Alternatively, Siess \& Livio (1999) have proposed that the high rotation rates, chromospheric activity, and lithium abundance seen in a small fraction of $\mathrm{G}$ and $\mathrm{K}$ giant stars can be explained by consumption of substellar companions. The accretion of a 1-2 Jovian mass planet from a decaying orbit onto an old main-sequence star might explain the anomalous observations described here.

E. J. G. thanks the personnel of KPNO, especially D. Willmarth, for courteous and professional assistance in the spectroscopic observations. We are grateful for the efforts of Lou Boyd and Don Epand at Fairborn Observatory. We thank Frank Fekel for useful discussions and for originally suggesting this collaboration. The research described in this paper was carried out in part by the Jet Propulsion Laboratory, California Institute of Technology, under contract with the National Aeronautics and Space Administration. Astronomy with automated telescopes at Tennessee State University is supported through NASA grants NCC5-96 and NCC5-228, which funds TSU's Center for Automated Space Science, and NSF grant HRD 97-06268, which funds TSU's Center for Systems Science Research.

\section{REFERENCES}

Benz, W., \& Mayor, M. 1981, A\&A, 93, 235

Binney, J., \& Tremaine, S. 1987, Galactic Dynamics (Princeton: Princeton Univ. Press)

Cameron, A. G. W., \& Fowler, W. A. 1971, ApJ, 164, 111

Duncan, D. K., et al. 1991, ApJS, 76, 383

Duquennoy, A., \& Mayor, M. 1991, A\&A, 248, 485

Eggen, O. J. 1998, AJ, 116, 1810

Fekel, F. C. 1997, PASP, 109, 514

Fekel, F. C., \& Balachandran, S. 1993, ApJ, 403, 708

Flower, P. J. 1996, ApJ, 469, 355

Gaidos, E. J. 1998, PASP, 110, 1259

Giannuzzi, M. A. 1979, A\&A, 77, 214

Güdel, M., Guinan, E. F., \& Skinner, S. L. 1997, ApJ, 483, 947

Henry, G. W. 1995, in ASP Conf. Ser. 79, Robotic Telescopes: Current Capabilities, Present Developments, and Future Prospects for Automated Astronomy, ed. G. W. Henry \& J. A. Eaton (San Francisco: ASP), 44 . 1999, PẢSP, 111, 845

Horne, J. H., \& Baliunas, S. L. 1986, ApJ, 302, 757

Jeffries, R. D. 1995, MNRAS, 273, 559

Johnson, D. R. H., \& Soderblom, D. R. 1987, AJ, 93, 864

Kastner, J. H., Zuckerman, B., Weintraub, D. A., \& Forveille, T. 1997, Science, 277, 67

King, J. R., Deliyannis, C. P., \& Boesgaard, A. M. 1996, AJ, 112, 2839

Lachaume, R., Dominik, C., Lanz, T., \& Habing, H. J. 1999, A\&A, 348, 897

Messina, S., Guinan, E. F., Lanza, A. F., \& Ambruster, C. 1999, A\&A, 347, 249
Moore, J. H., \& Paddock, G. F. 1950, ApJ, 112, 48

Noyes, R. W., Hartmann, L. W., Baliunas, S. L., Duncan, D. K., \& Vaughan, A. H. 1984, ApJ, 279, 763

Pasquini, L., Liu, Q., \& Pallavicini, R. 1994, A\&A, 287, 191

Perryman, M. A. C., et al. 1998, A\&A, 331, 81

. 1997, A\&A, 323, L49

Radick, R. R., Lockwood, G. W., Skiff, B. A., \& Baliunas, S. L. 1998, ApJS, 118,239

Saar, S. H., \& Osten, R. A. 1997, MNRAS, 284, 803

Siess, L., \& Livio, M. 1999, MNRAS, 308, 1133

Simon, T., \& Drake, S. A. 1989, ApJ, 346, 303

Soderblom, D. R. 1983, ApJS, 53, 1

Soderblom, D. R., \& Clements, S. D. 1988, AJ, 95, 580

Soderblom, D. R., Jones, B. F., Balachandran, S., Stauffer, J. R., Duncan,

D. K., Fedele, S. B., \& Hudon, J. D. 1993, AJ, 106, 1059

Soderblom, D. R., \& Mayor, M. 1993, AJ, 105, 226

Soderblom, D. R., Oey, M. S., Johnson, D. R. H., \& Stone, R. P. S. 1990, AJ, 99, 595

Sterzik, M. F., Alcalá, J. M., Covino, E., \& Petr, M. G. 1999, A\&A, 346, L41

Vaughan, A. H., Preston, G. W., \& Wilson, O. C. 1978, PASP, 90, 267

Webb, R. A., Zuckerman, B., Platais, I., Patience, J., White, R. J., Schwartz, M. J., \& McCarthy, C. 1999, ApJ, 512, L63

Wilson, O. C. 1978, ApJ, 226, 379 